\title{
Implementación de la APs en una zona urbana marginal de Cali: una experiencia de alianzas locales para enfrentar determinantes sociales*
}

\section{Implementation of PHC in a Marginal Urban Area of Cali: An Experience of Local Alliances to Face Social Determinants}

\section{Implementação da APs em uma zona urbana marginal de Cali: experiência de parcerias locais para enfrentar determinantes sociais}

Fecha de recepción: 28-01-15 Fecha de aceptación: 20-04-15 Disponible en línea: 30-06-15

doi: 10.11144/Javeriana.rgyps18-28.iazu

Como citar este artículo:

Holguín-Ruiz J, Aristizábal-Grisales JC, Murillo-Hurtado E, Acosta-Ramírez N. Implementación de la APS en una zona urbana marginal de Cali: una experiencia de alianzas locales para enfrentar determinantes sociales. Rev. Gerenc. Polít. Salud. 2015; 14(28): 109-122. http://dx.doi.org/10.11144/Javeriana. rgyps18-28.iazu

\author{
Jorge Holguín-Ruiz** \\ Juan Carlos Aristizábal-Grisales*** \\ Edwin Murillo-Hurtado**** \\ Naydú Acosta-Ramírez*****
}

Resultados de un proyecto desarrollado en el marco de la investigación titulada Caracterización de las condiciones de salud, Barrio "Potrero Grande-Somos Pacifico-Sector 9", comuna 21, Santiago de Cali, la cual recibió financiamiento de la Pontificia Universidad Javeriana-Cali (puJ-Cali). Inició en el 2010 y cubrió hasta el 2014, liderado por la PUJ-Cali, con el apoyo de la Fundación Paz y Bien, la Caja de Compensación Familiar Comfandi (convenio docente asistencial 473, renovado en el 2012 y vigente a la fecha), la Empresa Social de Estado (ESE) Oriente (convenio docente asistencial firmado en el 2009). Hallazgos y logros preliminares presentados en el V Foro Internacional de Cuidado Primario Ambulatorio, organizado por Javesalud el 14 de noviembre del 2014, con el apoyo de la PUj en Bogotá.

** Máster en Epidemiología, especialista en Otorrinolaringología, Médico. Profesor del Departamento de Salud Pública y Epidemiología de la Pontificia Universidad Javeriana-Cali. Correo electrónico: jholguinr@javerianacali.edu.co. Dirección: Calle 18 \# 118-250. Cali (V), Colombia.

*** Máster en Epidemiología, estadístico, profesor de la Pontificia Universidad Javeriana-Cali, Departamento de Salud Pública y Epidemiología. Correo electrónico: jcaristizabal@javerianacali.edu.co.

**** Especialista en Epidemiología, licenciado en Ciencias Sociales, Analyst Inter Center for Healthcare Innovation of Chicago-Illinois, Estados Unidos. Correo electrónico: edwin.j.murillo@gmail.com.

***** Doctora en Ciencias en Salud Pública, máster en Administración de Salud, médica, profesora de la Pontificia Universidad Javeriana-Cali, Departamento de Salud Pública y Epidemiología. Correo electrónico: naydu.acosta@ javerianacali.edu.co. 


\section{Resumen}

Debido a la persistencia de problemáticas sociales y de salud complejas en zonas marginales de una gran urbe, se desarrolló una intervención mediante un proyecto de investigación-acción con participación de academia, actores institucionales y comunitarios, en el marco de la Atención Primaria en Salud (APS). La primera fase tuvo como objetivo realizar el diagnóstico de las condiciones de salud y la segunda fase fue la acción o intervención, ambas guiadas con el enfoque de determinantes sociales. El diagnóstico fue un estudio de prevalencia que reveló, entre otros resultados, hacinamiento crítico y bajas coberturas de vacunación. Los logros fueron la socialización del diagnóstico, la concertación de acciones, las alianzas locales y la realización de intervenciones concretas de mejoramiento como: jornadas de promoción y prevención dirigidas a reducir y controlar los factores de riesgo identificados, acceso a medicamentos efectivos y oportunos, control de brotes, aumento de coberturas de vacunación y fortalecimiento de la acción interinstitucional.

Palabras clave: Atención Primaria en Salud; diagnóstico de la situación de salud; investigación aplicada; determinantes sociales de la salud; Colombia

\section{Abstract}

Due to the persistence of complex social and health problems in the marginal areas of a large city, we developed an intervention by means of a research-action project with the participation of the academy, institutional and community actors, under the frame of Primary Healthcare. The purpose of the first phase was to perform a diagnosis of the health conditions, and the purpose of the second phase was the action or intervention, both guided by a focus on social determinants. The diagnosis was a prevalence study which revealed critical overcrowding and low vaccination rates, among other results. We accomplished the socialization of the diagnosis, agreeing upon actions, local alliances, and the performance of concrete improvement interventions such as: promotion and prevention fairs aimed at lowering and controlling the identified risk factors; timely access to effective drugs; outbreak control; increase in the coverage of vaccinations; and strengthening the inter-institutional actions.

Keywords: primary healthcare; health situation diagnosis; applied research: social determinants of health; Colombia

\section{Resumo}

Devido à persistência de complexas problemáticas sociais e de saúde em zonas marginais de uma grande urbe, desenvolveu-se uma intervenção mediante projeto de pesquisa-ação com participação de academia, atores institucionais e comunitários, no âmbito da Atenção Primária à Saúde. A primeira fase teve como objetivo realizar o diagnóstico das condições de saúde e a segunda fase a ação ou intervenção, ambas as duas guiadas com enfoque de determinantes sociais. O diagnóstico foi um estudo de prevalência que desvendou, entre outros resultados, a superlotação crítica e as baixas coberturas vacinais. As conquistas foram a socialização do diagnóstico, concertação de ações, parcerias locais e realização de intervenções concretas de melhoramento como: oficinas de promoção e prevenção dirigidas a reduzir e controlar fatores de risco identificados, acesso a medicamentos efetivos e oportunos, controle de surtos, aumento de coberturas de vacinação e fortalecimento da ação interinstitucional.

Palavras-chave: Atenção Primária à Saúde; Diagnóstico da Situação da Saúde; Pesquisa aplicada; Determinantes Sociais da Saúde; Colômbia 


\section{Introducción}

La ciudad de Cali es una de las tres grandes urbes de Colombia. En su zona oriental, y de manera específica la comuna 21, existen áreas marginales con una serie de condiciones de vulnerabilidad social, relacionada con características tales como bajo nivel socioeconómico de la población - en su mayoría afrodescendientes- - y alta densidad poblacional. Adicionalmente, cerca de la mitad de la población son menores de edad con baja incorporación al sistema educativo (1-3).

En esta comuna se destaca por la complejidad de sus problemáticas sociales el barrio Potrero Grande (también denominado Somos Pacífico), el cual fue creado por decisión gubernamental y está conformado principalmente por habitantes provenientes de diferentes áreas de invasión y otros sectores marginales de la ciudad, tales como los sectores de Ladera, Aguablanca, la ronda del río Cauca y El Calvario, ubicado este último en el centro de la ciudad. Estas características de poblamiento se convierten en un factor determinante para la generación de conflictos e inestabilidad social, más aun teniendo en cuenta que no han sido llevados a cabo procesos de desarrollo social que faciliten la convivencia entre personas de procedencias tan disimiles y con amplia diversidad en materia étnica y sociocultural como lo son los habitantes de la costa del Pacífico, el interior de los departamentos de Cauca y Nariño, la zona andina e incluso el oriente del país (1).

En infraestructura, en Potrero Grande se construyó un importante equipamiento social y de servicios como ha sido la constante en casi todos los programas de vivienda de interés social de la comuna 21 , representados en un colegio con capacidad para más de dos mil estudiantes y un Centro de Desarrollo Infantil (CDI) con capacidad para quinientos niños, financiado y orientado por el Instituto
Colombiano de Bienestar Familiar (ICBF) y gestionado por un operador privado: la Caja de Compensación Familiar "Comfandi". También cuenta con dos instituciones de salud, un amplio y moderno centro de salud $\mathrm{y}$ un puesto de salud, ambos pertenecientes a la Red de Salud del Oriente "ESE Oriente" y cuenta con los servicios públicos básicos como son: acueducto, energía, alcantarillado y red telefónica, además de un trazado de vías debidamente pavimentadas con andenes adecuados y señalización en el interior del sector, que garantizan la conexión directa con la ciudad, lo que permite la oferta y presencia de diferentes medios de transporte público.

A pesar de ese equipamiento físico, el barrio tiene problemas de infraestructura de vivienda por el tamaño del área habitacional construida, la cual es de $40 \mathrm{~m}^{2}$. En esa área se encuentran dos habitaciones, distribuidas una el primer piso y otra en el segundo, un baño, escalera y un patio, sin espacios adecuadamente definidos para la cocina, la sala y el comedor, lo cual genera condiciones de hacinamiento con espacios pequeños de no más de $5 \mathrm{~m}^{2}$ por habitación y donde deben ubicarse algunas familias numerosas (de entre cinco y doce integrantes, aproximadamente).

Estos aspectos sin duda se convierten en factores determinantes de las condiciones de salud de los habitantes del sector, propician la aparición y el desarrollo de problemáticas de salud y socialmente relevantes en el interior de las familias, como la gestación y proliferación de enfermedades infecciosas y otros problemas de salud pública como el abuso sexual y la violencia intrafamiliar.

Dentro de los factores de desarrollo local en Potrero Grande se presentan marcadas dificultades, como las siguientes (1-3):

- Las condiciones de ingreso son precarias, ya que el estrato socioeconómico 
más común es el 1 (estrato moda), con el $80,7 \%$ de sus viviendas en este nivel, y solo el $19,3 \%$ en el estrato 2.

- El desarrollo comunitario también es precario, lo cual se evidencia en la presencia de conflictos entre líderes y miembros de diferentes organizaciones no gubernamentales, comunitarias y dignatarios de las juntas de acción comunal, y de estos con representantes de los organismos del Estado y ong que intentan ingresar a la zona.

- La convivencia es afectada por la violencia social y urbana. En los tres primeros trimestres del 2006 en la comuna se presentaron el 4,7\% de los homicidios de la ciudad, el equivalente a 77 homicidios por cada 100000 habitantes, valor este por encima de la tasa de homicidios de Cali. Las tasas de hurtos también están entre las más altas de la ciudad.

Con este contexto problemático y considerando que desde el 2011 se define normativamente para el país la estrategia de Atención Primaria en Salud (APS) (4), se decide realizar un proyecto de investigación-acción en un sector del barrio Potrero Grande de la comuna 21 de la ciudad de Cali, en el marco de la APs renovada (5), buscando propiciar la participación activa de diversos sectores sociales y la comunidad tanto en la evaluación como en los procesos de concertación y gestión, mediante el trabajo conjunto de diversos actores institucionales y los líderes, las organizaciones comunitarias y habitantes del sector, con el propósito de lograr tanto la visibilización de las problemáticas como la búsqueda conjunta de soluciones.

En este artículo se presenta el desarrollo y los resultados de ese proyecto, el cual se planteó

112 en dos fases. La primera fue la investigación, que tuvo como objetivo realizar el diagnósti- co de las condiciones de salud del territorio, a través de la identificación y caracterización de los factores de riesgo y los determinantes de la salud y la enfermedad de la población a nivel individual, familiar y comunitario, con la finalidad de posibilitar el diseño, la implementación, la evaluación, el seguimiento, la gestión y la concertación de políticas públicas, planes, programas y proyectos de mejoramiento de las condiciones de salud de los habitantes de este sector.

La segunda fase fue la intervención o acción, guiada también por el enfoque de determinantes sociales, que como los define la oms corresponden tanto a factores asociados a las condiciones de vida, el entorno sociocultural y las políticas definidas por el Estado, como también lo son la provisión de los servicios de salud, el medio ambiente y los estilos de vida, siendo estos últimos muy relevantes en salud pública al constituirse en factores modificables mediante intervenciones de promoción y prevención (6).

Este proyecto fue impulsado y dirigido por el Departamento de Salud Pública y Epidemiología de la Facultad de Ciencias de la Salud de la Pontificia Universidad Javeriana sede Cali (PUJ-Cali), como respuesta al modelo pedagógico de formación de los profesionales en Medicina, el cual involucra el diseño de una estrategia de intervención en la comunidad para posibilitar el crecimiento, la formación y el aprendizaje de los educandos, y como aporte sustancial desde el compromiso en responsabilidad social de la Universidad, que propende por el desarrollo de actividades y procesos encaminados al mejoramiento de las condiciones de vida de un conjunto poblacional mediante la conjunción de los aportes que se puedan hacer desde la academia y demás sectores involucrados.

Este proyecto fue liderado por la PUJ-Cali y se desarrolló en alianza con la Fundación Paz 
y Bien (institución de apoyo para los contactos con la comunidad en la zona), así como también con Comfandi (organización facilitadora de la intervención) y la ESE Oriente como entidad gubernamental rectora de los programas y proyectos de promoción de la salud y prevención de la enfermedad que se orientan desde el Estado.

\section{Materiales y métodos}

En la fase inicial se realizó un estudio observacional descriptivo de corte transversal que incluyó toda la población del sector 9 del barrio Potrero Grande, área seleccionada por las instituciones de la alianza institucional mencionada, con los criterios de alta vulnerabilidad socioeconómica y social. Este barrio, como ya se dijo, se encuentra ubicado en la comuna 21, al extremo oriental de la ciudad. La comuna tiene una extensión que corresponde aproximadamente al $4 \%$ del área total del municipio de Santiago Cali $(1,2)$.

Las fuentes de información son primarias, considerando como unidad de observación a los individuos y sus respectivas familias. Las variables que fueron observadas corresponden a los siguientes aspectos: condiciones sociodemográficas, conformación familiar, estado de la vivienda, morbilidad por grupos de edad, aspectos de salud sexual y reproductiva, planificación familiar, problemas sociales y factores de riesgo para la salud.

\section{Recolección y procesamiento de información}

Para la recolección de la información fue utilizada la tarjeta familiar del Sistema de Información de Base Comunitaria para Atención Primaria en Salud (Sicaps 2000), del Centro de Investigaciones Multidisciplinarias para el Desarrollo (Cimder), adscrito a la Universidad del Valle. Este instrumento está dirigido a caracterizar el estado de salud y sus factores condicionantes, amparado en un concepto amplio de salud que incluye los determinantes sociales (7). Una vez aplicadas y recolectadas las tarjetas familiares, estas fueron digitadas en un programa especializado complementario del sistema Sicaps, el cual generó las respectivas bases de datos. El análisis incluyó procedimientos de estadística descriptiva para el cálculo de proporciones, indicadores de tendencia central y variabilidad, y de posición, de acuerdo con el tipo de variable analizada.

Esta investigación fue aprobada por el Comité de Ética de la PUJ-Cali y, de acuerdo con los lineamientos normativos de Colombia para aspectos éticos de la investigación en seres humanos, Resolución 8430 de 1993 (8), la clasificación de este estudio corresponde a una investigación sin riesgo, ya que se emplean técnicas y métodos de investigación sin ninguna intervención o modificación intencionada de las variables biológicas, fisiológicas, sicológicas o sociales de los individuos que participan en el estudio.

El trabajo de campo se realizó entre diciembre del 2010 y el primer semestre del año 2011 con personal entrenado. Para estandarizar y garantizar la calidad y confiabilidad de la información se elaboró un plan de trabajo, el cual contenía los instructivos y mapas con codificación de las manzanas del sector, un cronograma con los respectivos responsables de cada una de las actividades y el procedimiento de articulación con los representantes de la comunidad del sector 9 .

Paralelamente, como estrategia para garantizar la calidad de los datos, se crearon círculos de calidad, comenzando por el personal de encuestadores, quienes se encargaron de entregar las tarjetas completamente diligenciadas; posteriormente, estas fueron revisadas por supervisores, quienes a través de la selección de una muestra aleatoria de tarjetas evaluaron la calidad de estas; finalmente, los 
digitadores realizaron la evaluación crítica para verificar la validez de la información. A la par con el desarrollo del trabajo de campo, se elaboró una base de datos con los criterios de calidad, cobertura y oportunidad de la información. Esta información fue llevada a formatos TXT (Excel) y luego se procesó en el programa estadístico sPss.

\section{Resultados}

De acuerdo con los resultados presentados en la tabla 1, en total fueron encuestadas 412 familias, que correspondieron a un total de 1593 personas. Con base en esta información, en este sector una familia está compuestas por cuatro personas, aproximadamente.

\section{Características sociodemográficas}

Los encuestados en promedio tenían 23,7 años, IC 95\% (22,9-24,6); en mayor proporción fueron mujeres $(55 \%)$ y la raza predominante fue afrodescendiente, con más de las tres cuartas partes de los encuestados identificados en esta categoría $(75,3 \%)$.

El régimen de afiliación al sistema general de seguridad social en salud reportado con mayor frecuencia fue el subsidiado, con un valor de $79,7 \%$ del total de los encuestados,

Tabla 1. Variables sociodemográficas del sector 9, Potrero Grande, Cali-2011

\begin{tabular}{|c|c|c|c|}
\hline Variable & Indicador & Valor & $\%$ \\
\hline \multirow{3}{*}{ Población } & Total familias & 412 & $\mathrm{Na}$ \\
\hline & Total personas & 1593 & $\mathrm{Na}$ \\
\hline & Personas por familia & 3,9 & $\mathrm{Na}$ \\
\hline \multirow{4}{*}{ Edad (años) } & Promedio & 23,7 & $\mathrm{Na}$ \\
\hline & Desviación estándar & 17,6 & $\mathrm{Na}$ \\
\hline & IC 95\% media (límite inferior) & 22,9 & $\mathrm{Na}$ \\
\hline & IC 95\% media (límite superior) & 24,6 & $\mathrm{Na}$ \\
\hline \multirow{2}{*}{ Sexo } & Mujeres & 876 & $55,0 \%$ \\
\hline & Hombres & 717 & $45,0 \%$ \\
\hline \multirow{3}{*}{ Grupos étnicos } & Afrodescendiente & 1199 & $75,3 \%$ \\
\hline & Mestizo & 358 & $22,5 \%$ \\
\hline & Indígena & 36 & $2,3 \%$ \\
\hline \multirow{4}{*}{ Afiliación sGSSS } & Subsidiado & 1269 & $79,7 \%$ \\
\hline & Pobre no asegurado & 230 & $14,4 \%$ \\
\hline & Contributivo & 92 & $5,8 \%$ \\
\hline & Otro & 2 & $0,1 \%$ \\
\hline \multirow{6}{*}{ Escolaridad } & No sabe leer ni escribir & 81 & $5,1 \%$ \\
\hline & Nunca fueron a la escuela pero saben leer y escribir & 760 & $47,7 \%$ \\
\hline & Básica primaria & 384 & $24,1 \%$ \\
\hline & Secundaria & 349 & $21,9 \%$ \\
\hline & Superior & 6 & $0,4 \%$ \\
\hline & No recuerdan el grado & 13 & $0,8 \%$ \\
\hline
\end{tabular}

Fuente: elaboración propia de los autores, cálculos del estudio 
seguido de $14,4 \%$ que expresaron no tener ningún aseguramiento en salud (pobre no asegurado); solamente el 5,8\% manifestó estar afiliado al régimen contributivo.

En cuanto a la escolaridad, se encontró que más de las tres cuartas partes del total de los encuestados reportaron como máximo nivel educativo la básica primaria, y de ellos $70 \%$ no han tenido acceso a ningún tipo de formación académica; adicionalmente, se encontró que menos de una cuarta parte de los encuestados manifestó haber realizado estudios de secundaria.

En la figura 1 se puede observar que hasta los catorce años de edad, en donde se encuentra aproximadamente el $38 \%$ de los encuestados, la proporción entre hombres y mujeres es prácticamente igual, en tanto que $48 \%$ de los encuestados se encuentran en el rango de 15 a 45 años y $14 \%$ en el de mayores de 45 años. En estos dos rangos de edad se encuentra una notable mayor proporción de mujeres, es decir, que en el rango de la población económicamente activa de la zona, la proporción de mujeres prácticamente supera el $60 \%$.

En la figura 2, en donde se presenta la pirámide poblacional, se puede observar la brecha existente entre la cantidad de hombres y la de mujeres, de manera marcada entre los veinte y los sesenta años, situación mencionada anteriormente. Dichas diferencias son más pronunciadas entre los más jóvenes. También se identifica una gran proporción de adolescentes y una relevante cantidad de menores de cinco años.

\section{Características generales de las viviendas, los hogares y las familias}

En el 99\% de los hogares del sector 9 son utilizados materiales adecuados para la construcción de paredes, pisos y techos. De la misma manera, en este sector se presentan coberturas totales y similares en lo relacionado con el acceso a los servicios públicos tales como electricidad, agua potable y alcantarillado.

En cuanto a las condiciones de la familia dentro de la vivienda, se destaca que fue reportado hacinamiento crítico en 58,67\% de las familias encuestadas, ya que se identificaron familias de hasta doce personas habitando una misma vivienda. En este sentido, es necesario tener en cuenta que este hacinamiento es favorecido por el área construida de la vivienda, la cual se encuentra alrededor de los $40 \mathrm{~m}^{2}$.

Con relación a la tenencia de la vivienda, $76 \%$ de las familias viven en casa propia. Del total de las familias encuestadas, en el $61,4 \%$ se encontró a la mujer como cabeza de familia.

La composición familiar se distribuyó en las siguientes categorías: hogares monoparentales $8,5 \%$, hogares nucleares $62,6 \%$ y hogares de familia extensa/compuesta $28,9 \%$.

Con respecto a los riesgos presentes en la vivienda, fue reportada la presencia de roedores en $56 \%$ de las viviendas, exposición a fumadores en $22 \%$, consumo de bebidas alcohólicas en $18,3 \%$ y consumo de sustancias psicoactivas en $2,2 \%$.

En cuanto al desarrollo de tejido social y familiar en la zona, en el 29,7\% de los hogares se informó que alguien del núcleo familiar participaba en actividades de tipo comunitario. El $82,6 \%$ califican sus relaciones familiares como buenas, en tanto que el $76,9 \%$ de las familias comparten actividades que permiten la unión familiar y en el 14,8\% de los hogares encuestados se informó de la realización de actividades productivas en su interior. 
Jorge Holguín-Ruiz • Juan Carlos Aristizábal-Grisales • Edwin Murillo-Hurtado • Naydú Acosta-RAmíREZ

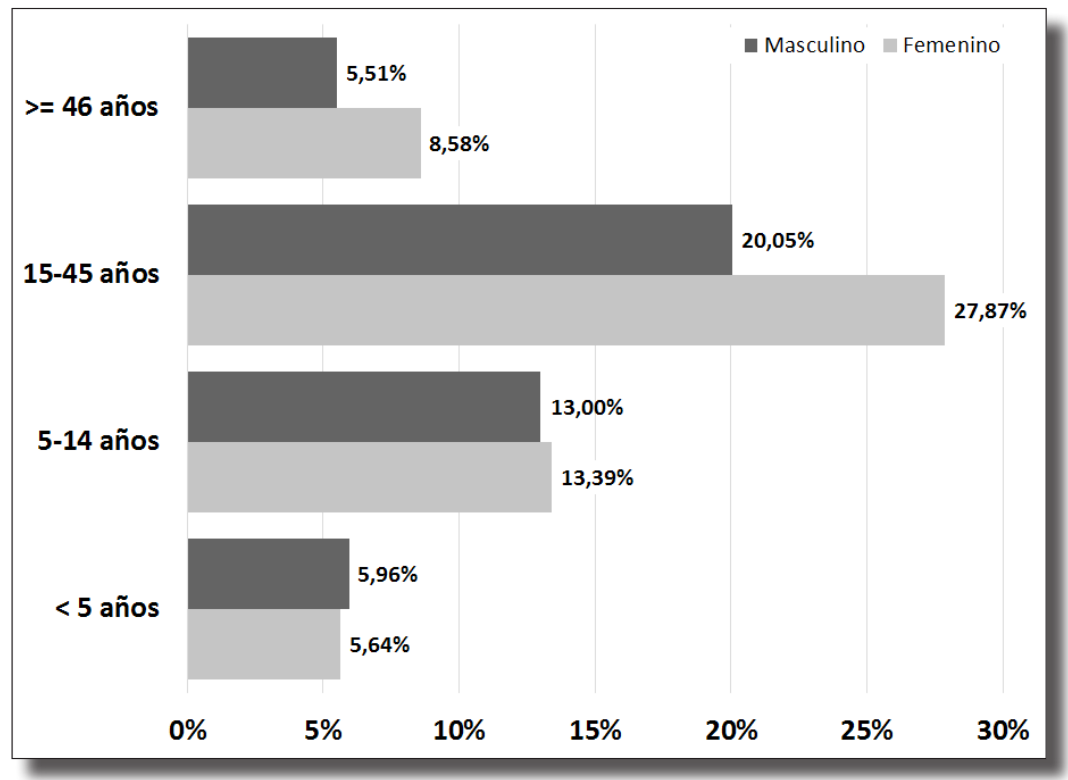

Figura 1. Distribución por rango de edad y sexo, sector 9, Potrero Grande, Cali-2011

Fuente: elaboración propia de los autores, cálculos del estudio

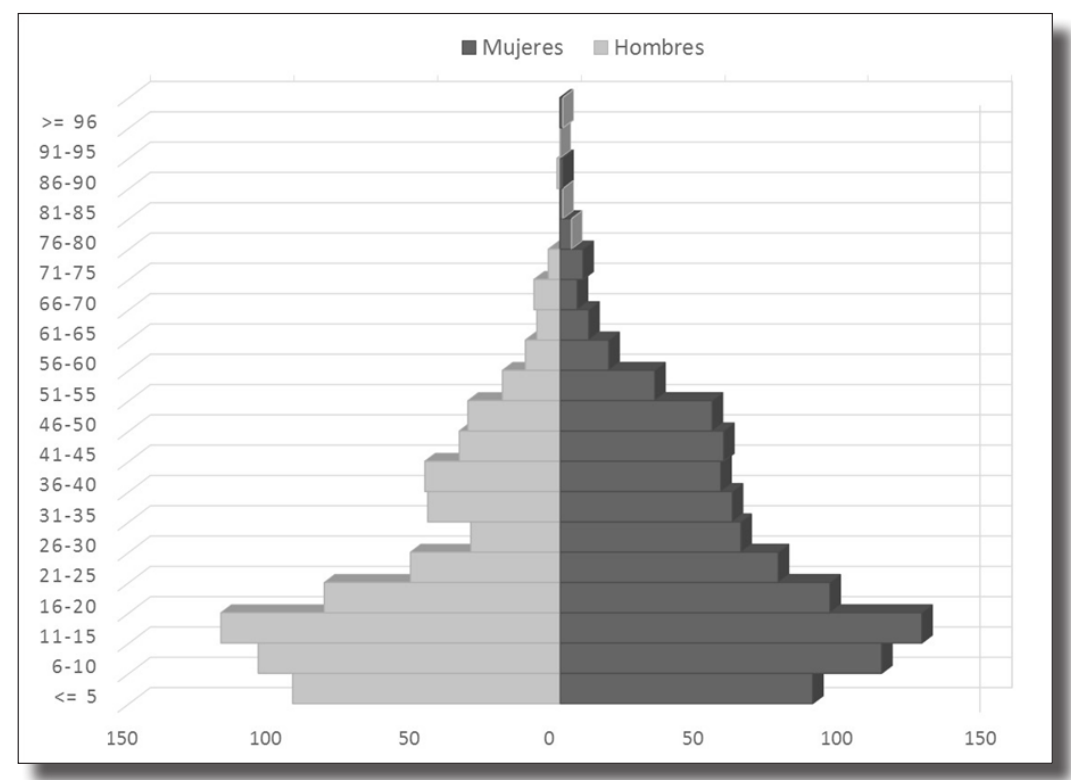

Figura 2. Pirámide poblacional, sector 9, Potrero Grande, Cali-2011

Fuente: elaboración propia de los autores, cálculos del estudio 
Descripción de las condiciones de salud y riesgos asociados

A la pregunta relacionada con la percepción del estado de salud individual, del total de los encuestados, ninguno manifestó percibir su estado de salud como excelente, $57 \%$ refirieron un buen estado de salud, $27 \%$ manifestaron que su estado de salud era regular y $3 \%$ informaron que la percepción de su salud se encontraba entre mala y muy mala.

Con respecto a la vacunación en menores de un año, llaman la atención las bajas coberturas en todos los biológicos estudiados -fiebre amarilla, BCG, DPT tres dosis, polio tres dosis, hepatitis B tres dosis, HIB, SRP, influenza dos dosis y neumococo dos dosis- (se encuentran coberturas entre 13 y $81,5 \%$ ); adicionalmente, solo fue reportada la tenencia de carné de vacunación en $75 \%$ del total de los menores de un año y de ellos solo $50 \%$ asistieron a los controles de crecimiento y desarrollo.

En los niños y niñas entre uno y cuatro años, se reportó que $31,5 \%$ de ellos tenían su esquema de vacunación incompleto. En este grupo no asistieron a control de crecimiento y desarrollo el $31 \%$ y presentan alteraciones en la talla/edad, peso/edad, peso/talla hasta en un $30 \%$. Asimismo, refieren alteraciones visuales $11,6 \%$, auditivas $13 \%$ y de conducta 19,2\%. En los niños de cinco a catorce años fueron reportadas alteraciones visuales en $17 \%$ de los niños, auditivas en $8,5 \%$ y de conducta en $11,2 \%$.

En el sector 9 también fue identificado un $17,4 \%$ de embarazo en adolescentes, mientras que en mayores de 35 años se presentó una proporción de gestantes de 8,7\%. Del total de las veintitrés embarazadas reportadas en el sector, realizaron revisión odontológica $38 \%$, recibieron hierro y ácido fólico $84 \%$ y no reportan hábito de fumar, pero consumen bebidas alcohólicas el $5 \%$.

En el grupo de hombres y mujeres de 10 a 52 años se encontró que $64,9 \%$ de los hombres y $34,6 \%$ de mujeres no realizan planificación familiar. De manera general, hombres y mujeres manifestaron como las principales razones para no planificar: $38,5 \%$ porque no tenían compañero, $25 \%$ no habían tomado aún la decisión y $8,8 \%$ dejan la responsabilidad a la pareja. En el grupo de adolescentes $53,7 \%$ no planifican, debido a que no tienen compañero, en tanto que $7,4 \%$ corresponde a mujeres que no lo hacen porque se encuentran en gestación. Los hombres y mujeres adolescentes reportan que fuman en 3,7 y $2,3 \%$, respectivamente, y $37,3 \%$ de ellos realizan actividad física adecuada.

\section{Intervención en la zona: Jornadas de salud Integrales}

Con base en los resultados de la investigación inicial descrita se desarrollaron diferentes actividades que corresponden a la fase de intervención-acción en la zona de desarrollo del proyecto. La primera actividad fue la socialización de la información del diagnóstico y la definición de intervenciones en jornadas de salud a nivel del barrio en el sector $9 \mathrm{y}$ en el Centro de Desarrollo Infantil (CDI) El Paraíso, donde se han focalizado acciones como proyecto de responsabilidad social de la PUJCali. El cDI está ubicado en el barrio Potrero Grande de la comuna 21, es una modalidad de atención a la primera infancia enmarcada en la Estrategia de Cero a Siempre, cuya misión es promover el desarrollo infantil a través de una atención integral a quinientos niños y niñas menores de cinco años de edad en condiciones de vulnerabilidad.

La jornada de socialización de resultados de la encuesta fue presentada en agosto del 2011, con asistencia de líderes del sector y 
la comunidad en general, así como de las máximas autoridades de la Universidad, con asistencia del señor rector, el decano, directivas y profesores del Departamento de Salud Pública y Epidemiología de la Facultad de Ciencias de la Salud.

Posteriormente, en cuanto a las actividades del proceso de atención integral, desde el segundo semestre del 2011 se cuenta con una alianza con el CDI en el marco del convenio docente asistencial entre la caja de compensación familiar Comfandi y la Facultad de Ciencias de la Salud de la Pontificia Universidad Javeriana-Cali, para el desarrollo de prácticas formativas de la carrera de Medicina. Mediante esta alianza se han desarrollado estrategias de intervención de APS, dirigidas al mejoramiento de las condiciones de salud de los niños vinculados al CDI, además de apoyar la formación de las familias y los agentes educativos en promoción de la salud y prevención de la enfermedad, las cuales son herramientas básicas dentro del esquema de garantía de derechos de los niños.

Esta decisión surge del análisis de la encuesta en la que se destaca que la población de menores de cinco años del sector 9 de Potrero Grande corresponde al 11,6\% (184 niños). Con respecto al componente de vacunación en menores de cinco años, se encuentra poca tenencia de carné de vacunación en menores de un año $(75 \%)$ y bajas coberturas en todos los biológicos estudiados; además, no reportaron tener el carné de vacunación en niños de uno a cuatro años.

Basados en esos resultados, se planteó la necesidad de realizar una intervención con el enfoque de APs para actualizar los esquemas de vacunación de todos los niños asistentes al CDI, los cuales provienen de todos los sectores del barrio. Además, se decidió fortalecer a padres y cuidadores en prácticas claves de promoción de la salud infantil que promueve la Organización Panamericana de la Salud (ops) (9).

Con esa finalidad se organizó la primera jornada comunitaria en el 2011, con visita domiciliaria realizada por docentes y estudiantes de los semestres I al IV de la PUJCali, beneficiando a más de 460 hogares del sector 9. Para las actividades los estudiantes se distribuyeron en parejas que visitaron en promedio seis viviendas de ese sector. El objetivo era informar a la comunidad sobre los riesgos en salud más importantes a los que estaba expuesta y cómo prevenirlos. Asimismo, los estudiantes realizaron, bajo supervisión docente, las remisiones pertinentes de la población al centro de salud del sector, y de los niños sin esquema de vacunación completo al puesto de vacunación móvil, en donde el equipo de salud de la ESE Oriente y de la Secretaría de Salud Municipal actualizaron el esquema de inmunizaciones de los niños y niñas menores de cinco años; adicionalmente, se aplicaron vacunas a adultos, especialmente contra sarampión y rubeola.

A partir de esa experiencia, para la cátedra de Salud Pública II (centrada en niños y adolescentes), el Departamento de Salud Pública y Epidemiología de la PUJ-Cali focalizó como centro de práctica comunitaria de los estudiantes de IV semestre de la carrera de Medicina el CDI El Paraíso. Los estudiantes realizan su práctica con menores de cinco años, con observación dirigida por docentes, centrándose en la identificación e intervención de aspectos de la salud y la enfermedad y condiciones socioeconómicas, entre otros.

Las denominadas Jornadas de Salud Integrales se realizan siguiendo la distribución académica asignada de dos grupos de estudiantes por semestre, con cupo promedio de veinticinco estudiantes por grupo, desarrolladas en dos días diferentes en los meses de mayo y noviembre, para un total de once 
jornadas desarrolladas actualmente, hasta la culminación del primer semestre académico del año 2014, en las cuales han participando en promedio más de 250 estudiantes de la carrera de Medicina. Estas jornadas se realizan en alianza con diversas instituciones de la zona. Así, se ha contado con el apoyo de Comfandi (Subdirección de Salud), el Instituto Colombiano de Bienestar Familiar, la Secretaría de Salud Municipal de Cali y la ESE Oriente.

\section{Actividades y beneficiarios}

De acuerdo con la base institucional de registros de usuarios (quinientos niños y niñas), la distribución por sexo de la población menor de cinco años que asiste al CDI El Paraíso corresponde principalmente a niños, con un $57 \%$.

Se reporta que el $23 \%$ de las familias beneficiarias del CDI están en situación de desplazamiento.
La pertenencia étnica informada en el CDI corresponde en su mayoría a población afrocolombiana, en un $66,8 \%$, cifra más baja que la correspondiente al sector 9 del Barrio Potrero Grande (75,3\%) (ver figura 3).

De las Jornadas de Salud Infantil Integral en el CDI, en el balance del primer semestre del 2014, se encuentran como logros:

1. Actualizar el esquema de vacunación del Plan Ampliado de Inmunización (PAI) de quinientos niños menores de cinco años, con más de mil dosis de vacunas del PAI aplicadas (principalmente fiebre amarilla, influenza, MMR, polio y DPT), además de la articulación intersectorial, con lo cual se benefició con vacuna no PAI contra la varicela a todos los niños asistentes (ochocientas dosis en dos jornadas independientes de la Secretaría de Salud Pública y la ESE Oriente). Todo esto ha impactado en la salud y el bienestar de los niños, con disminución

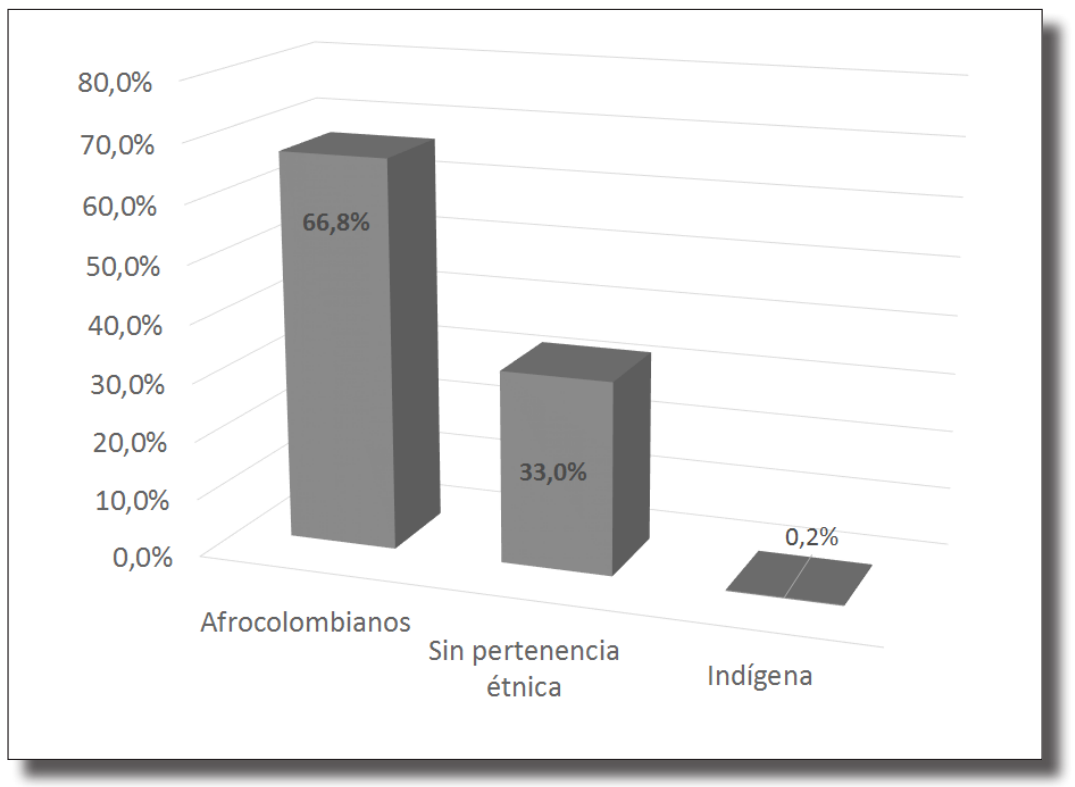

Figura 3. Pertenencia étnica de la población beneficiaria del cdi El Paraíso, Cali 2014

Fuente: elaboración propia de los autores, cálculos del estudio 
a cero en los brotes de varicela y disminución de ausentismo y complicaciones de esta patología.

2. Aproximadamente trescientas actividades de salud oral, correspondientes en su mayoría a higiene oral.

3. Aproximadamente 150 actividades de valoración de agudeza visual.

4. Más de 250 niños valorados en sus condiciones de salud durante las prácticas formativas de los estudiantes de Medicina.

5. Trescientas personas, que corresponden a padres, acudientes y agentes educativas con formación en las dieciocho prácticas clave de salud infantil, centrados en la atención integral de las enfermedades de la infancia (AIEPI) e incluyendo nutrición como tema solicitado por la comunidad.

6. Más de 650 hombres y mujeres (incluyen personas asistentes al centro de salud de Potrero Grande en las primeras cuatro jornadas) participantes en estrategias de información, educación y comunicación en aspectos de la salud sexual y reproductiva, prevención de infecciones de transmisión sexual (ITS), VIH-SIDA y educación en planificación familiar.

7. Más de 120 consultas médicas realizadas durante las diferentes jornadas en la institución de primera infancia (con la participación de médicos de la ESE Oriente, Comfandi y la Pontificia Universidad Javeriana Cali). Como la principal causa de consulta se encontraron las infecciones respiratorias agudas.

8. Actualización de certificados médicos y orales de todos los niños y niñas asistentes.
9. Seguimiento con antropometría a trescientos niños y niñas menores de cinco años.

10. Donación de más de doce grupos de materiales didácticos de educación en salud orientada a las madres, para prevenir los problemas más frecuentes de la infancia según las dieciocho prácticas clave de salud infantil- AIEPI comunitario, salud sexual y reproductiva, prevención de ITS, VIH-SIDA y educación en planificación familiar.

\section{Discusión y conclusiones}

Desde la promulgación de la APS en 1978 en Alma Ata, se ha reconocido que para lograr mayores resultados en la salud poblacional se requieren procesos de intervención acordes a las necesidades poblacionales, y que para la sostenibilidad deberían ajustarse a los recursos locales (10). En este estudio se evidencia y se concluye cómo la alianza de la academia, las instituciones de bienestar social y de salud y la comunidad permite desarrollar esos aspectos.

Un eje crítico inicial fue el diagnóstico de las condiciones de salud, una herramienta fundamental de evaluación y planeación que está enfocada en identificar, caracterizar y analizar el comportamiento de los factores determinantes de la salud y predisponentes de la enfermedad a nivel de una comunidad, localidad o región, otorgando a la comunidad, al Estado y a los demás actores de la sociedad elementos analíticos para la formulación e implementación de políticas públicas saludables, programas y proyectos de promoción de la salud, prevención y control de los factores de riesgo generadores de enfermedad en la población. 
La aplicación del Sicaps tiene a la comunidad como su punto de partida y finalización (7). Los datos surgen de la comunidad, básicamente a través de las visitas domiciliarias, pero también deberían regresar a esta en forma de intervenciones tales como acciones de salud o bienestar tangibles por la comunidad, al constituirse como información que se transforma en necesidades poblacionales para ser abordadas en la toma de decisiones locales. Los diferentes instrumentos y subsistemas que conforman el Sicaps permiten la vigilancia individual, familiar y colectiva del estado de salud y de la presencia de las enfermedades en la población. Una fortaleza de este estudio es que la aplicación de la encuesta Sicaps cubrió toda la población de un sector, con lo cual se maximiza el poder de estimación de algunas prevalencias de eventos de salud encontradas.

En términos generales, los diagnósticos de la situación de salud de una comunidad, localidad o región no solo son la herramienta básica para la planeación y definición de políticas públicas saludables y programas y proyectos en salud, sino también el mecanismo básico por excelencia para la identificación de los factores determinantes de la salud y los factores de riesgo para las enfermedades.

En consecuencia, basados en los resultados de la encuesta en la segunda fase del proyecto antes descrito, se desarrolló la fase intervención-acción en el marco de APS, con énfasis en acciones de promoción y prevención, intervenciones extramulares, participación comunitaria y alianzas intersectoriales que propendan por enfrentar los determinantes sociales específicos.

El componente de promoción de la salud fue dirigido a la sensibilización y formación a padres de familia y agentes educativos en las prácticas clave de salud infantil de la estrategia AIEPI (9). Se realizó un énfasis de acuerdo con los principios y lineamientos de la APs en los hallazgos de problemáticas más frecuentes, encontrados en las Jornadas de Salud Integral y en estudios previos en la zona (11), tales como enfermedades diarreicas agudas, enfermedad febril, dengue e infecciones respiratorias agudas, incluyendo las medidas preventivas y de salud ambiental.

Otro componente de la segunda fase de intervención del proyecto se centró en acciones de prevención y como logro relevante se destaca que con las jornadas de salud integrales se mejoró el acceso a medicamentos efectivos y oportunos a partir de donaciones. También, se logró en el periodo de intervención el control de brotes de varicela, debido a la vacunación de todos los niños del CDI en el año 2013 y la actualización 2014, con lo cual paralelamente se apoyó en el cumplimiento a los estándares de calidad por parte del CDI referentes a la garantía de derechos de los niños en salud. La integración de los componentes de promoción y prevención primaria y actuar sobre factores de riesgo en la comunidad permite el mejoramiento de la salud, al contribuir a la implementación de intervenciones más efectivas en salud, como lo han evidenciado investigaciones de revisión en APS en otros contextos (12).

Además, es relevante destacar las alianzas locales y el fortalecimiento interinstitucional entre la Universidad Javeriana, el CDI El Paraíso, la Red de Salud ESE Oriente, Comfandi y el ICBF, lo cual deriva en el mejoramiento de las condiciones de salud de los niños vinculados al CDI El Paraíso, al realizar alianzas para acciones conjuntas como las descritas, que incluyen educación en salud, detección temprana de condiciones y enfermedades en los niños y remisión oportuna. Las alianzas intra e intersectoriales son críticas en países en desarrollo como Colombia, donde la conjunción de intereses permite hacer un uso eficiente de los escasos recursos disponibles 
para enfocarlos y adecuarlos a las necesidades poblacionales prioritarias, como lo han evidenciado diversos estudios previos (13).

Por otro lado, en la renovación de la APs otros autores y estudios destacan el rol crítico del talento humano, el cual debería contar con competencias que incluyan conocimientos, habilidades y destrezas para transcender el enfoque biomédico tradicional, centrado en el individuo y la atención hospitalaria de la enfermedad, y lograr un cambio hacia un enfoque familiar y comunitario que parta del reconocimiento de territorios y poblaciones con necesidades particulares, para el desarrollo de acciones integrales, efectivas y oportunas $(14,15)$. Así, en este proyecto se muestra y se concluye cómo el involucrar estudiantes de programas universitarios como Medicina, en prácticas comunitarias con trabajo de campo en barrios marginales, se constituye en una estrategia útil para el fortalecimiento de la formación de los futuros profesionales en esas competencias mencionadas.

\section{Agradecimientos}

A la dirección del Departamento de Salud Pública y Epidemiología, a cargo durante la ejecución inicial del proyecto del Dr. Mauricio Serra-Tamayo y posteriormente por la doctora Marcela Arrivillaga-Quintero; a los docentes de la cátedra de Salud Pública II, doctora Mérida Rodríguez (periodo 2011-2013), doctora Claudia Criollo (periodo 2013-2014); a la doctora Rosa Elena Vásquez, coordinadora del CDI El Paraíso y a todo el personal que labora en CDI; funcionarios de la ESE Oriente-Secretaría de Salud de Cali y personal que labora en el Centro de Salud de Potrero Grande.

\section{Referencias bibliográficas}

1. Alonso JC, Solano MA, Vera R, Gallego AI. Una mirada descriptiva a las comunas de Cali. Cali:
Cienfi, Universidad Icesi, Departamento Administrativo de Planeación Municipal; 2007.

2. Municipio de Santiago de Cali, Secretaría de Salud Municipal. Análisis Situacional Integrado de Salud de Cali (AsIs). Cali: Secretaría de Salud Municipal; 2012.

3. Municipio de Santiago de Cali, Secretaría de Salud Municipal. Análisis de la Situación Integral de Salud (ASIS): Documento de análisis de situación de salud con el modelo de los determinantes sociales de salud. Cali: Secretaría de Salud Municipal; 2013.

4. Congreso de la República, República de Colombia. Ley 1438, enero 19 del 2011. Por medio de la cual se reforma el Sistema General de Seguridad Social en Salud (sGsss).

5. Macinko J, Montenegro H, Nebot C, Etienne C. La renovación de la atención primaria de salud en las Américas. Rev Panam Salud Pública. 2007; 21(2-3): 73-84.

6. Álvarez Castaño LS. Los determinantes sociales de la salud: más allá de los factores de riesgo. Revista Gerencia y Políticas de Salud. 2009; 8 (17): 69-79.

7. Zapata-Ossa HJ, Cubides-Munévar AM, López MC, Pinzón-Gómez EM, Filigrana-Villegas PA, Cassiani-Miranda CA. Muestreo por conglomerados en encuestas poblacionales. Rev Salud Pública. 2011; 13 (1): 141-51.

8. Ministerio de Salud, República de Colombia. Resolución 8430 de 1993, Octubre 4. Por la cual se establecen las normas científico-técnicas y administrativas para la investigación en salud.

9. Organización Panamericana de la Salud (ops), Ministerio de Salud y Protección Social. Guía para la atención y la consejería de la niñez en la familia. AIEPI Comunitario. Bogotá: OPS; 2010.

10. oms. Conferencia Internacional de Atención Primaria de Salud, Declaración de Alma Ata. Alma-Ata, Unión Soviética, 6 al 12 de septiembre de 1978.

11. Holguín Ruiz JA. Evaluación del impacto del botadero a cielo abierto de navarro en la prevalencia de síntomas de infección respiratoria aguda en niños de 1 a 5 años residentes en su área de influencia [tesis de maestría]. Santiago de Cali: Maestría en Epidemiología, Escuela de Salud Pública, Universidad del Valle; 2006.

12. Da Silva LS, Cotta RM, Rosa CO. Health Promotion and Primary Prevention Strategies to Fight Chronic Disease: A Systematic Review. Rev Panam Salud Pública. 2013 Nov; 34 (5): 343-50.

13. Ramírez Acosta N, Ruiz Pollard, Romero Vega R, Labonté R. Comprehensive Primary Health Care in South America: Contexts, Achievements and Policy Implications. Cad. Saúde Pública. 2011; 27 (10): 1875-90.

14. Organización Panamericana de la Salud (OPs). Serie La renovación de la atención primaria de salud de las Américas. No 1. Sistemas de salud basados en la atención primaria de la salud: estrategias para el desarrollo de los equipos de APs. Washington D.C.: OPS; 2008.

15. Rey-Gamero AC, Acosta-Ramírez N. El enfoque de competencias para los equipos de Atención Primaria en Salud. Una revisión de literatura. Revista Gerencia y Políticas de Salud, 2013; 12 (25): 28-39. 